\title{
Editorial
}

\section{Trends in lung cancer mortality}

Carcinoma of the bronchus is the most common cancer in British men, while in women only cancers of the breast, large bowel, and skin have a higher incidence. Despite improvements in treatment the fatality rate remains high, and the tumour currently causes close to 35000 deaths a year in England and Wales. ${ }^{1}$ Four fifths of the deaths occur in men. Like most other Western countries, Britain has experienced an epidemic of lung cancer over the course of this century. ${ }^{2}$ Can this epidemic be attributed entirely to smoking, or have other factors such as air pollution contributed to the trends? What course is the epidemic likely to follow in years to come? Clues to the answers to these questions emerge from a more detailed examination of mortality patterns.

When the incidence of a disease is changing it is important to distinguish any differences between the trends in different age groups. Figure 1 shows age specific mortality rates from lung cancer in England and Wales plotted against year of death. While mortality is still increasing in the elderly, death rates in young people are now falling. Furthermore, this decline in mortality began first in the youngest age groups.

The pattern becomes clearer if the data are rearranged as in figure 2. Year of death has been replaced on the horizontal axis by year of birth (calculated by subtracting age at death from year of death), so that each vertical set of points represents the mortality rate at different ages of persons born during a 10 year period surrounding a central year of birth. Male mortality at each age reaches a peak in the generation born during 1901-16. In women a similar pattern is emerging, but with the highest death rates occurring among those born during the 1920s.

Care is needed in the interpretation of statistics such as these. Apparent increases in mortality may arise from improvements in diagnosis (for example, through the introduction of diagnostic radiology and bronchoscopy) or from changes in diagnostic fashion. At the same time, improvements in treatment may reduce mortality while incidence rates remain unchanged. By and large, artefacts of this kind would be expected to affect both sexes and all age

Address for reprint requests: Dr D Coggon, MRC Environmental Epidemiology Unit, Southampton General Hospital, Southampton SO9 4XY.

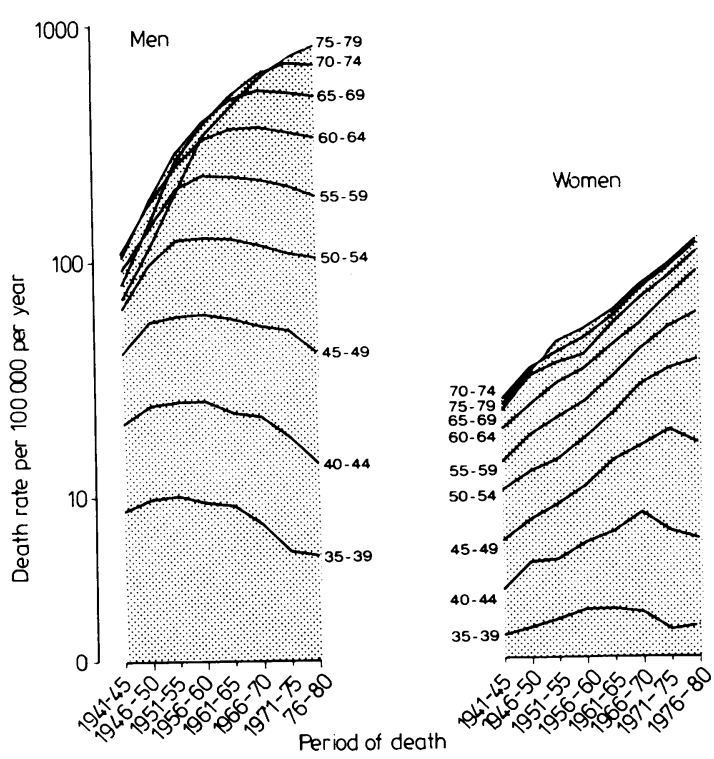

Fig 1 Age specific mortality from lung cancer in England and Wales during 1941-80 plotted against period of death.

groups simultaneously. That the trends observed for lung cancer mortality are different in men and women, and show such a noticeable generation effect is strong evidence that they reflect real changes in incidence. The carcinogen responsible for the epidemic must start to act by early adult life since its effects are manifest below the age of 40 , and exposure to it must have been maximal in those generations with the highest mortality.

The difference between the sexes in the timing of the epidemic suggests that general environmental factors such as air pollution are unlikely to be the major underlying cause. The rise in mortality is much more consistent with the effects of smoking, with the delayed peak in women explained by the fact that smoking became popular among women later than among men. There has been no substantial decrease in the tobacco consumption of recent generations to account for the falling incidence of lung cancer in young persons, ${ }^{3}$ and it seems likely that the latter is instead attributable to a reduction in the tar content of cigarettes and the introduction of filter tips. ${ }^{4}$ Recent studies have indicated that the 


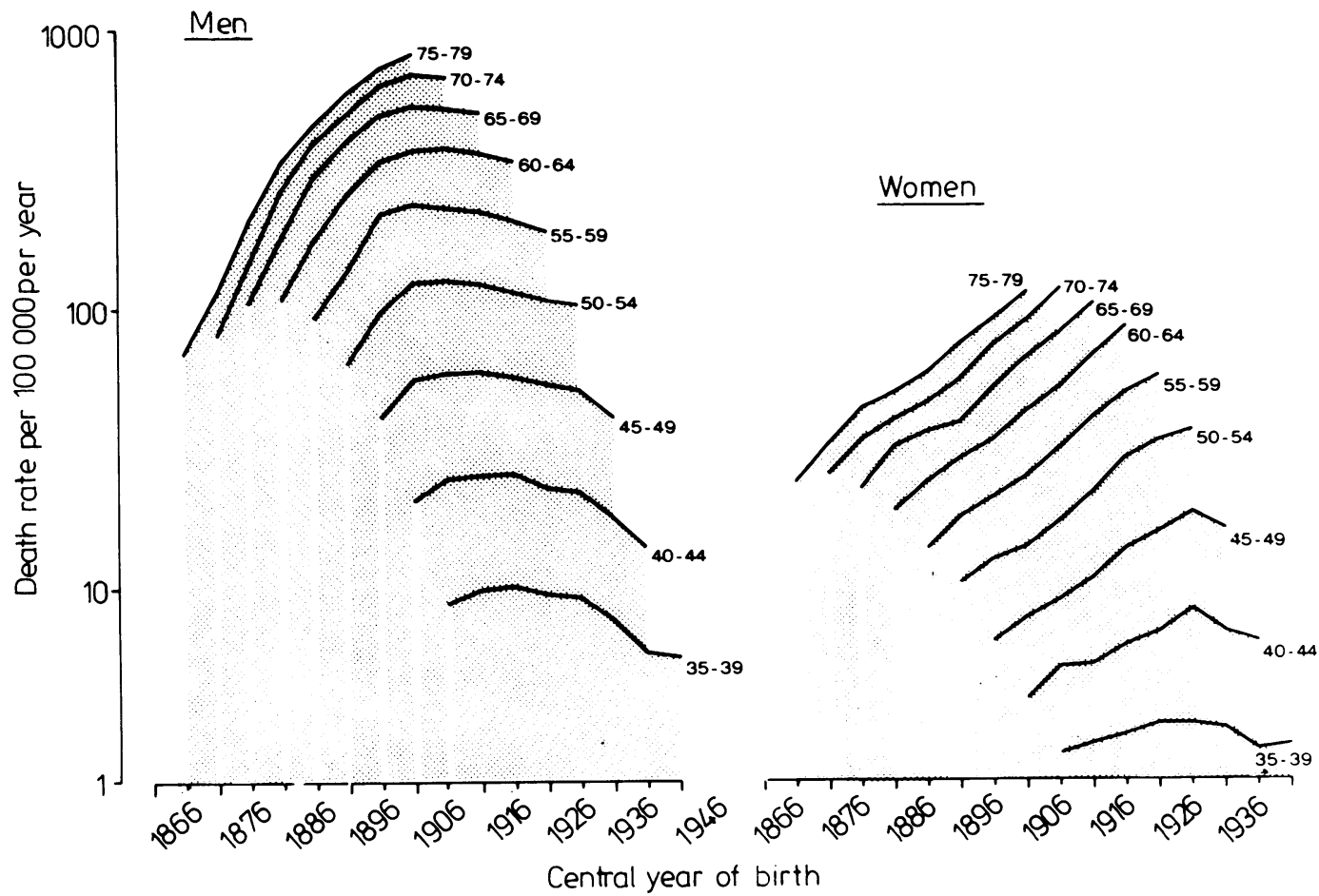

Fig 2 Age specific mortality from lung cancer in England and Wales during 1941-80 plotted against central year of birth.

risk of lung cancer is lower in long term users of filter cigarettes than in those smoking the non-filter variety. ${ }^{5}$

No other known cause of lung cancer explains the recent trends in mortality as well as smoking does. Nevertheless, other factors may have influenced the behaviour of the disease to a lesser degree. In particular, Adelstein has suggested that the reduction in air pollution by smoke from domestic fires (which began after the first world war and was accelerated in the mid-1950s by the Clean Air Act of 1956) may have contributed to the decline in lung cancer deaths among young people. ${ }^{6}$

The strongest evidence that air pollution causes carcinoma of the bronchus lies in the consistent urban-rural gradient in incidence and mortality. When differences in age and sex are taken into account the mortality from lung cancer in conurbations of England and Wales is 1.5 times that in rural districts. ${ }^{7}$ A similar excess of mortality in urban areas has been observed in the United States. ${ }^{8}$ Urban air contains several known lung carcinogens, including asbestos and arsenic as well as polycyclic aromatic hydrocarbons from the incomplete combustion of fossil fuel; but the importance of such pollutants is difficult to establish because the exposure of individuals, particularly in the past, cannot be reliably estimated.

Undoubtedly factors other than pollution contribute to the urban excess of lung cancer. Smoking is more common among the residents of cities than in rural populations. Attempts have been made to 3 allow for differences in tobacco consumption in comparisons of urban and rural incidence, ${ }^{910}$ but they have not taken account of factors such as duration of smoking and age at which smoking commenced. Doll and Peto have pointed out that when a new habit is becoming established in society it is likely to be taken up first in cities. ${ }^{11}$ For this reason $\sigma$ city dwellers who smoke are likely to have done so $N$ for longer than those living in the country.

Another reason for the higher incidence of lung cancer in urban areas is the increased risk of the disease in certain occupations. A list of industrial products and processes which are known or sus- $\stackrel{\Phi}{\oplus}$ pected causes of bronchial carcinoma is given in the table. In terms of the number of cases produced asbestos is by far the most important, and may $\mathbb{\mathbb { D }}$ account for as many as $5 \%$ of deaths from lung cancer in the United States. ${ }^{11}$ Apart from haematite 
Industrial products and processes known to cause or suspected of causing lung cancer

Known causes

Nickel refining

Underground haematite mining (with exposure to radon)

Arsenic and arsenic compounds

Asbestos

Bis(chloromethyl)ether and technical grade chloromethyl methyl ether

Chromium and certain chromium compounds

Mustard gas

Soots, tars, and oils

Suspected causes

Acrylonitrile

Beryllium and beryllium compounds

Dimethyl sulphate

mining the hazards are all more prevalent in industrial towns and cities than in the country, and so contribute to the urban-rural gradient in incidence.

Because of difficulties in measuring individual exposure to pollutants, and at the same time taking account of confounding factors such as smoking and occupation, epidemiologists have resorted to indirect estimates of the impact of air pollution on lung cancer mortality. Extrapolation from the risks associated with much higher levels of exposure to the same carcinogens, such as are encountered in the work place, suggests that the proportion of deaths from lung cancer attributable to pollution is very much smaller than the proportion due to smoking. ${ }^{11}$

When all age groups are considered together, mortality from carcinoma of the bronchus is still on the increase in England and Wales. The death rate in men is now stabilising but in women it continues to rise, and will do so for at least 10 years if current trends are maintained. This is because most deaths from lung cancer occur in the older age groups, where the incidence is still increasing. In the long term mortality can be expected to fall, though the extent of this decline is difficult to estimate. There is a long way to go before we reach the low incidence of 50 years ago, and while smoking remains popular it is unlikely that this will be achieved.

$$
\begin{array}{r}
\text { D COGGON } \\
\text { ED ACHESON } \\
\text { Medical Research Council Epidemiology Unit } \\
\text { Southampton General Hospital } \\
\text { Southampton }
\end{array}
$$

\section{References}

' Office of Population Censuses and Surveys. Mortality statistics: cause, 1980. London: HMSO, 1982.

${ }^{2}$ Alderson M. International mortality statistics. London: Macmillan Press, 1981.

${ }^{3}$ Lee PN, ed. Statistics of smoking in the United Kingdom. 7th ed. London: Tobacco Research Council, 1976. (Research Paper 1.)

${ }^{4}$ Wald N, Doll R, Copeland G. Trends in tar, nicotine and carbon monoxide yields of UK cigarettes manufactured since 1934. Br Med J 1981;282:763-5.

${ }^{5}$ Wynder EL, Stellman SD. Comparative epidemiology of tobacco-related cancers. Cancer Res 1977;37:460822.

${ }^{6}$ Adelstein AM. Encouragement from recent statistics. In: Raven RW, ed. Outlook on cancer. New York: Plenum Press, 1977: 255-75.

7 Office of Population Censuses and Surveys. Area mortality: decennial supplement 1969-73 England and Wales. London: HMSO, 1981.

${ }^{8}$ Blot WJ, Fraumeni JF jun. Geographic patterns of lung cancer: industrial correlations. Am J Epidemiol 1976;103:539-50.

9 Stocks P, Campbell MJ. Lung cancer death rates among non-smokers and pipe and cigarette smokers. An evaluation in relation to air pollution by benzpyrene and other substances. Br Med J 1955;ii:923-39.

${ }^{10}$ Dean G. Lung cancer and bronchitis in Northern Ireland 1960-1962. Br Med J 1966;i:1506-14.

${ }^{11}$ Doll R, Peto R. The causes of cancer: quantitative estimates of avoidable risks of cancer in the United States today. J Natl Cancer Inst 1981;66:1192-308. 